\title{
Basic Facts about Biosimilars
}

\author{
Michał Nowicki \\ Department of Nephrology, Hypertension and Kidney Transplantation, Medical University of Łódź, Łódź, Poland
}

\section{Key Words}

Biotechnological medicines · Epoetin • Biosimilars •

Biologics, follow-on • Immunogenicity

\begin{abstract}
Biotechnological drugs have become an essential part of modern pharmacotherapy and are expected to reach a $50 \%$ share in the pharmaceutical market in the next few years. The expiry of patent protection for many original biotechnological medicines has led to the development of what are called biosimilars or follow-on biologics. Biosimilars attempt to copy the original technology leading to the production of innovative biotechnological medicines to obtain a product which is similar to the original one. The first two biosimilars have recently been approved in the European Union and one application was rejected. Many more biosimilars will likely see approval in the near future. Our experience with biosimilars has been very limited to date and long-term safety data including immunogenicity are not available. Although biosimilars will likely lower the cost of modern therapies there are issues which have to be discussed at this stage among physicians regarding in particular the differences between biosimilars and generics of the classical chemical drugs, need for appropriate regulations as well as identification of potential problems with biosimilars. Other specific problems which will also be addressed in this review are safety of biosimilars, pharmacovigilance, automatic substitution, naming and labeling/prescription rules.
\end{abstract}

Copyright $\odot 2007$ S. Karger AG, Basel

\begin{tabular}{ll}
\hline KARGER & $\oplus$ 2007 S. Karger AG, Basel \\
Fax +4161306 1234 $34-4096 / 07 / 0305-0267 \$ 23.50 / 0$ \\
$\begin{array}{l}\text { E-Mail karger@karger.ch } \\
\text { www.karger.com }\end{array}$ & $\begin{array}{l}\text { Accessible online at: } \\
\text { www.karger.com } / \mathrm{kbr}\end{array}$
\end{tabular}

\section{What Are Pharmaceutical Proteins and What Is Their Role in Medicine?}

Biotechnological medicines are medicinal products of biotechnological origin that contain proteins derived from DNA technology and hybridoma techniques [1]. The biotechnologies use living organisms such as plant and animal cells, bacteria, viruses and yeasts for the production of medicines. Biotechnological medicines extend our abilities to fight diseases, including many which in the past had been recognized as incurable, e.g. orphan diseases. Therefore biotechnological medicines, which often replace or supplement a natural protein produced by the body, satisfy medical needs previously unmet by chemical medicines. The rapidly growing class of biotechnological medicines includes such biological factors as cytokines, hormones, clotting factors, monoclonal antibodies, vaccines and molecules used for cell/tissuebased therapies [2]. By definition biotechnological medicines are far more complex with regard to their structure than traditional chemical drugs, as is also their mode of action. While chemical agents usually affect one or a few processes in the living organism, the biotechnological molecule, e.g. recombinant interferon, interacts with nearly 100 genes which makes its exact mode of action really difficult to predict and explore. It is estimated that so far almost 500 million patients worldwide have been helped by biotechnological medicines [3]. By 2010 biopharmaceuticals will represent $50 \%$ of the pharmaceutical market, a leap from less than $20 \%$ in 2004 . According to the recent PhRMA Survey (2006) in addition to more

Prof. Dr med. Michał Nowicki

Department of Nephrology, Hypertension and Kidney Transplantation

Medical University of Łódź, Barlicki University Hospital

Kopcinskiego 22, PL-90-145 Łódź (Poland)

Tel. +48 426776 709, Fax +48 4264663 48, E-Mail nefro@wp.pl 
than 120 now existing, 418 new biopharmaceuticals are being developed in almost all areas of modern medicine but most in the field of oncology, infectious and autoimmune diseases and respiratory disorders [4].

Although chemical and biotechnological drugs are pharmaceutical products, they are completely different in terms of their manufacturing, structure and action. Chemical drugs have a well-defined structure whereas the structure of biotechnological medicines is much more complex and heterogeneous. This is reflected by the huge differences in a molecular weight of the molecules. For example the molecular weight of aspirin is $180 \mathrm{Da}$ whereas that of interferon- $\beta$ is $19,000 \mathrm{Da}$. Therefore the typical biologic drug molecule is $100-1,000$ times larger than that of a conventional (chemical) small molecule drug. The former also possesses a fragile 3-dimensional structure unlike the well-characterized 1-dimensional structure of chemical agents (chemical formula) which in most cases is easy to reproduce [3]. In contrast the structure of biotechnological medicines is very difficult to characterize because they are products of living cells and usually contain a mixture of different isoforms. For example recombinant erythropoietins (epoetins) differ in their carbohydrate structure and the number of sialic acid residues $[5,6]$. Chemical drugs are also much more stable whereas biological drugs are sensitive to changes in physical conditions which require not only strict control of the manufacturing process but also appropriate storage at pharmacies and by the patients. Most chemical drugs are taken orally but almost all biologics are injected or inhaled because, as proteins, they are very sensitive to enzymatic degradation in the gastrointestinal tract.

The manufacturing process leading to the production of a biotechnological medicine is also much more complex. A controlled manufacturing process is intrinsically important to biotechnological medicines. Manufacturing biotechnological medicines is complex, lengthy and expensive. Each manufacturer must make their own unique cell line which is a clone to a single cell. The cell line is constructed using a unique proprietary DNA expression vector. The characteristics of the incorporation of DNA is unique for each cell. The cell line is evaluated for product integrity, activity and overall quality. In addition to product quality, the cell line is chosen based on expected performance in manufacturing such as growth and viability. It is also clear that even minor variations could produce vastly different products since the process is extremely sensitive to changes in both manufacturing and production [7].

\section{What Are Biosimilars?}

The expiry of patent protection and regulatory data protection for certain biotechnological medicines has led to the development of what are called biosimilars [8]. Biosimilars attempt to copy the process which leads to the production of the original innovative biotechnological medicine. The term 'biosimilars' is in use in the European Union but in the US the term 'follow-on biologics' is much more popular.

It is important to state that biosimilars are not (bio)generics. Biosimilars are attempted copies of existing biological medicinal products or protein drugs. However, they are made with a different cell line and a different manufacturing and purification process, and the final product is not identical.

\section{Why Biosimilars Are Not (Bio)Generics}

Chemical drugs are in most cases relatively easy to reproduce as their structure is precisely defined and expressed by the chemical formula. The unique multi-dimensional structure of the proteins and, in consequence, their complicated mode of action are never fully reproducible. In fact biotechnological medicines, even with the same molecular weight and produced by the same type of cells or microorganisms, can possess different pharmacokinetic and pharmacodynamic properties [8]. This was well shown in the case of various $\alpha$-epoetins which are manufactured in many areas of the world breaking the patent-protection laws [6]. Such substances cannot be called biosimilars as they do not meet the regulatory approval standards.

In fact after the expiry of patent protection for original biotechnological products the first biosimilars entered the market in 2006 after having been approved by the European Agency for the Evaluation of Medicinal Products (EMEA). Those first biosimilars were Omnitrope (biosimilar to Genotropin) and Valtropin (biosimilar to $\mathrm{Hu}-$ matrope) $[9,10]$. It is of note, however, that EMEA has recently rejected the biosimilar Alpheon (interferon- $\alpha$ ) due to a higher number of side effects and more frequent recurrence of the disease in patients treated with Alpheon than with its reference product Roferon-A, and the lack of appropriate validation of the manufacturing process and the test used to evaluate a potential immunologic response to the drug [11]. Also multiple biosimilar products are under development for the European market and most of them are expected to be approved in the coming years. 
Fig. 1. The overview of the EMEA guidelines on biosimilars.

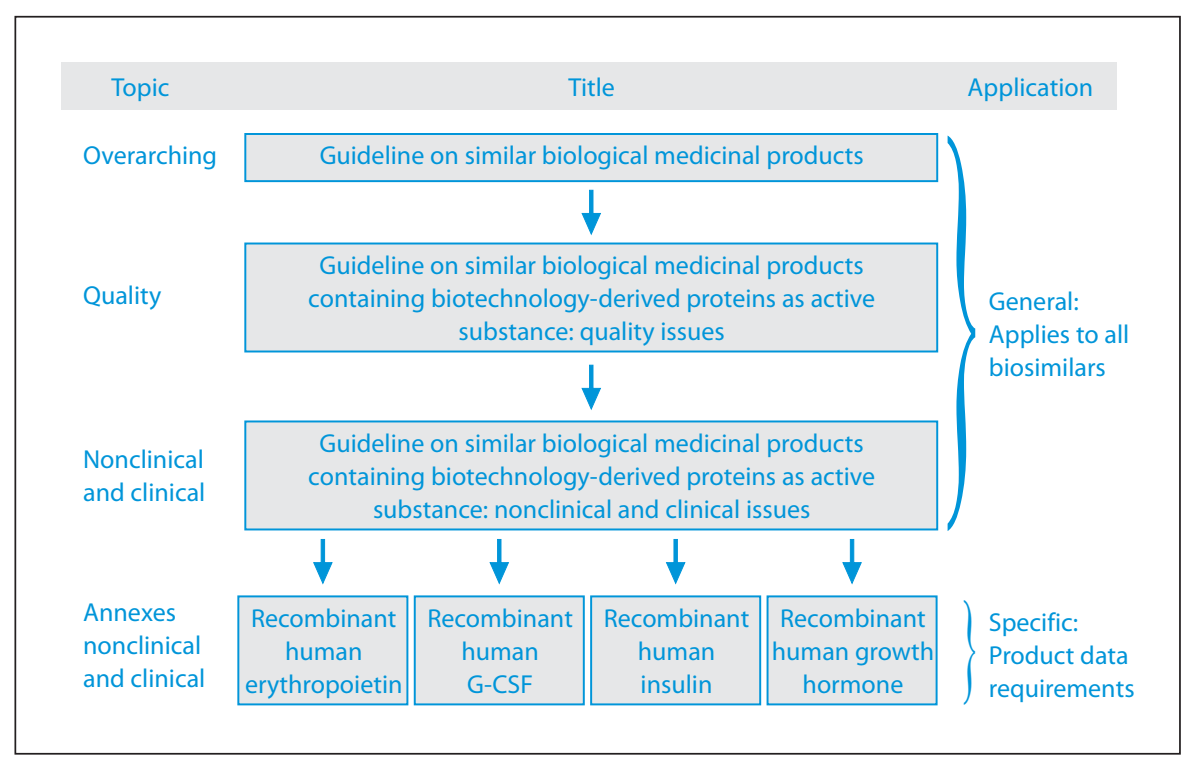

\section{Biosimilars - Legal and Regulatory Framework in Europe and the US}

The history of the development of regulatory laws in case of biogenerics is an exceptionally short one, but this is due to the fact that the issue virtually did not exist before expiry of patent protection for major biotechnological medicines on the market (i.e. epoetins, interferons, etc.) which took place mainly in the years 2004-2006. In fact the first recommendation for the approval of biogenerics was issued in 2004 by the European Parliament (European Directive 2004/27/EC). So far regulatory requirements for the approval of biosimilars have not been fully established and it is expected that the guidelines issued in 2005 by the EMEA (EMEA/CHMP/42832/2005) will undergo further changes in the near future [12]. Most importantly the guidelines emphasize the problems of the complexity of biotechnological products, the likely consequences of changes in the manufacturing process, and also the issues regarding the quality of the product, its safety and efficacy. Although these regulations are an important step in the right direction there is still uncertainty on the specific issue of the documentation necessary for an approval. Also the specificity of the different biotechnological medicines is not fully recognized. It is interesting that the European Union with its EMEA is a pioneer in the regulations on biosimilars since the agency for drug evaluation in the US, i.e. the Food and Drug Administration (FDA) is still working on the final version of the guidelines on follow-on biologics. In fact many patents for original biotechnological medicines will expire later in the US than in the EU countries (e.g. epoetin- $\alpha$ ).

Unfortunately, despite the publication of the aforementioned EMEA guidelines there are still no such regulations at the national levels in the member countries of the European Union. The only exception is France where in 2007 Parliament adopted legislation covering biosimilar medicines transposing the European Directive 2004/ 27/EC [13].

The most important issue of the EMEA guidelines is the notion that biotechnological medicines cannot simply be copied as has been the case with conventional chemical medicines $[12,14]$. However the guidelines accept minor differences in the active substance, such as variability in post-translational modifications. This recognition is crucial with respect to the safety of the new biotechnological products which will be approved in the near future. The overview of the EMEA guidelines on biosimilars is given in figure 1.

The other important part of the guidelines indicates that biosimilar manufacturers need to identify a single reference product and conduct tests to demonstrate biophysical similarity. It is also obligatory for the manufacturer to provide sufficient non-clinical (in vitro and in vivo pharmacodynamic and toxicological studies) and clinical data to demonstrate the clinical similarity to the reference product. In case of the biosimilar recombinant human erythropoietin for the treatment of renal anemia, the new product will have to be tested in at least two efficacy studies in chronic kidney disease patients including one titration study in epoetin-naïve patients (chronic 
kidney disease patients with subcutaneous administration of the drug) and one maintenance study in patients already treated with erythropoiesis-stimulating agents (chronic dialysis patients with intravenous administration of the product). Also a risk management/pharmacovigilance plan with attention paid to immunogenicity and potential rare serious adverse events should be presented before approval $[15,16]$. Furthermore an efficacy study with an appropriate indication is required if the reference product has multiple indications. Biosimilar manufacturers may extrapolate to other indications if the mechanism of action is the same and if appropriately justified.

Most importantly the guidance also requires immunogenicity data to be provided before approval. This is especially important if the recent lesson from the unexpected rise in the number of cases of pure red cell aplasia (PRCA) in patients treated for renal anemia with recombinant erythropoietin is considered (see below for details). As shown in figure 1, in the guidelines there are also product-specific annexes which provide details for certain biotechnological medicines such as epoetin [15], insulin, granulocyte colony-stimulating factor and human growth hormone.

\section{Identification of Potential Problems with Biosimilars?}

There are 4 major points to consider about biosimilars, i.e. safety, automatic substitution, naming and labeling/ prescription rules [8].

\section{Safety}

By definition biosimilars will only be similar but not identical to the product they seek to copy. In biotechnological medicine, each product has a unique safety profile dependent on its mechanism of action, unique manufacturing process, and composition (including byproducts and impurities). The best example to illustrate that the safety profile of the biosimilar will not be identical to that of the reference product is that the recently approved biosimilar growth hormone Valtropin has different precautions and warnings than its reference product Humatrope. This is a likely consequence of the different cell lines used to produce both drugs (yeasts in the case of Valtropin and Escherichia coli in the case of Humatrope) [17].

Immunogenicity is the unique safety issue of biotechnological medicines. All therapeutic proteins have the potential to induce antibody responses. Such a response may include both an initial response to therapeutic protein and later a broad response including endogenous proteins. In many cases, no clinical consequences are found but in rare cases an antibody-related reaction can be serious and even life-threatening. Unfortunately, the immunogenicity of biosimilars cannot be fully predicted using preclinical or non-human studies. For this reason clinical immunogenicity studies are required before approval, however robust pharmacovigilance still remains a critical part of the process.

There are various potential consequences of immunogenicity such as loss or enhancement of efficacy, neutralization of a native protein and general immune effects (allergy, anaphylaxis, serum sickness) [18]. The complication of epoetin therapy, i.e. PRCA, well known to nephrologists, was caused by the production of neutralizing antibodies directed against recombinant erythropoietin [19]. This complication was manifested by severe epoetin-resistant anemia which required blood transfusions, immunosuppressive treatment and eventually kidney transplantation. The story of PRCA was very instructive not only for nephrologists as its outbreak occurred more than 10 years after the introduction of epoetins on the market and was most likely caused by only a subtle change in the manufacturing process [20]. Taking into consideration the complexity of the action of therapeutic proteins and the fact that most of them are relatively new drugs, it is likely that we are not yet aware of all complications of their use (especially those that may develop after a longterm treatment). As mentioned before, to minimize the risk of such unexpected reactions an appropriate pharmacovigilance is mandatory. The issue of pharmacovigilance is not unique to biosimilars but without any doubt it has been highlighted and exaggerated by their arrival [21]. Pharmacovigilance should ensure the traceability of the products. Companies and regulatory agencies should distinguish one manufacturer's product from another. This is complex if biosimilars have the same international non-proprietary name (INN) as the innovator. Also adverse event reports are often incomplete. This holds especially true in cases of an automatic substitution. Uncontrolled substitution will confound accurate pharmacovigilance although it is obvious that occasional changes are inevitable or necessary in chronic therapy.

\section{Substitution}

The introduction of biosimilars raised many discussions whether the common notion of most practicing physicians that medicines with the same molecular structure are the same and therefore can be safely sub- 
stituted still holds true. In the case of generics of chemical drugs the substances are identical and the risk of substitution is expected to be low. Despite those claims such risk still exists. Most practicing physicians realize that not every patient can tolerate well every medicine based on the same chemical compound. Medical literature provides many reports showing that this holds especially true in the case of drugs with a narrow therapeutic window (e.g. anti-epileptics, anti-arrhythmics or cyclosporine). The principle behind substitution of the traditional chemical drugs is that the original drug and its generic are identical and have the same therapeutic effect. In consequence generics are approved following a demonstration of bioequivalence, thus excluding any clinically significant differences in bioavailability. In the case of biosimilars the same substitution rules cannot be applied since biotechnological medicines are not identical $[21,22]$.

The existing and future regulations should prevent inappropriate substitution [23]. Inappropriate substitution could occur in two ways, i.e. the pharmacist overrides the prescription of the physician or the pharmacist chooses a product from the same INN class. In the first case the physician prescribes a brand or a specific generic and the pharmacist overrides with an alternative 'generic' without consultation with the physician. In the second case the physician prescribes by INN but does not specify manufacturer and the pharmacist chooses one of the products with the same INN. In practice such choice is based on the price of the drug or personal experience. In both cases, however, substitution can occur without knowledge or consent of the physician. If it happened in the case of a biosimilar it would decrease the safety of the therapy. The future regulations regarding biosimilars should ensure the physicians that automatic generic substitution rules should not apply and any decisions to substitute one biotechnological medicine with another should be made with the knowledge and explicit prior consent of the physician [22].

\section{Naming}

The WHO INN system aims at identifying every medicinal product. This system is important for the clear identification, safe prescription and dispensing of medicines to patients, and for communication and exchange of information among health professionals and scientists worldwide. The INN is the 'technical' name for medicinal products. The generic version of chemical medicines are assigned the same name, as they are identical copies of the reference product. The WHO is currently deciding whether biosimilars should be assigned a different INN to that of the original biotechnological medicine [22].

\section{Labeling}

From all that is written above it is clear that labels of originator and biosimilar products should be different. Physicians, pharmacists and patients should be aware of the clinical data available to support an introduction of a medicine to therapy. A summary of product characteristics should be transparent and clear. Reference products should be defined. Also data for approval should be described. Furthermore unique safety data should be included and substitution advice should be provided.

\section{Conclusion}

Biotechnological medicines will become an important part of the future healthcare landscape. Biologics - and properly regulated biosimilars - will increasingly become available, will provide patients and doctors with alternative treatment options and most likely will make it possible to decrease the direct costs of therapies and increase their availability to the patients. Physicians should be aware that quality, safety and efficacy issues in the case of biotechnological medicines and biosimilars are key and much more complex than with traditional generics. Substitution rules between originator and biosimilar products must be different than in the case of generic substitutions as such products are never identical. Awareness of the differences between original biotechnological medicines and biosimilars is essential for the safety of the patients.

\section{Financial Disclosure Statement}

The author participated in the meetings sponsored by Roche and Amgen and received honoraria and consulting fees from Amgen. This paper was prepared without any commercial support. 


\section{References}

1 Schellekens H: Biopharmaceuticals and biosimilars, unraveling the complexity. EJHP Pract 2006;12:13.

72 Roger SD: Biosimilars: how similar or dissimilar they are. Nephrology 2006;11:341346.

-3 Karpusas M, Whitty A, Runkel L, Hochman P: The structure of human interferon-beta: implications for activity. Cell Mol Life Sci 1998;54:1203-1216.

4 http://www.phrma.org/files/Biotech\% 202006.pdf

5 Storring PL, Triplady RJ, Gaines Das RE, Rafferty B, Mistry YG: Lectin-binding assays for the isoforms of human erythropoietin: comparison of urinary and four recombinant erythropoietins. J Endocrinol 1996 150:401-412.

6 Schmidt CA, Ramos AS, da Silva JEP, Fronza M, Dalmora SL: Activity evaluation and characterization of recombinant human erythropoietin in pharmaceutical products. Arg Bras Endocrinol 2003;47:183-189.

$\checkmark 7$ Molowa DT, Mazanet R: The state of biopharmaceutical manufacturing. Biotechnol Ann Rev 2003;8:285-302.
8 Schellekens H: Follow-on biologics: challenges of the 'next generation'. Nephrol Dial Transplant 2005;20(suppl 4):iv31-iv36.

9 Nau JY: Omnitrope, first 'biosimilar' drug of the European Union. Rev Med Suisse 2006; 2:1206.

10 Burger J: The first approved biotech-generic Versicherungsmedizin 2006;58:190-191.

11 http://www.redorbit.com/news/health/ 557861/european_panel_snubs_biopartners_alpheon/index.html

12 http://www.emea.eu.int/pdfs/human/biosimilar/4283205en.pdf

13 http://www.europabio.org/articles/PR Biosimilars_070221_FINAL.pdf

14 Combe C, Tredree RL, Schellekens H: Biosimilar epoetins: an analysis based on recently implemented European Medicines Evaluation Agency guidelines on comparability of biopharmaceuticals proteins. Pharmacotherapy 2005;25:954-962.

15 http://www.emea.eu.int/pdfs/human/biosimilar/9452605en.pdf

16 Więcek A, Mikhail A: European regulatory guidelines for biosimilars. Nephrol Dial Transplant 2006;21(suppl 5):v17-v20.
17 http://www.ebe-biopharma.org/forcedownload.php?file $=/$ media $/$ biosimilars $/$ biosimilars_subsitutionpos.pdf

18 Thorpe R, Wadhwa M: Protein therapeutics and their immunity. EJHP Pract 2006;12: 17-19.

19 Casadevall N, Rossert J: Importance of biologic follow-ons: experience with EPO. Best Pract Res Clin Haematol 2005;18:381-387.

20 Lim LC: Acquired red cell aplasia in association with the use of recombinant erythropoietin in chronic renal failure. Hematology 2005; 10:255-259.

21 Locatelli F, Roger S: Comparative testing and pharmacovigilance of biosimilars. Nephrol Dial Transplant 2006;21(suppl 5):v13-v16.

22 http://www.ebe-biopharma.org/docs/pdf/ Biosims_EBEEFPIAPosition_Naming 7July2006.pdf

23 http://www.ebe-biopharma.org/forcedownload.php? file $=/$ media $/$ biosimilars $/$ biosimilars_subsitutionpos.pdf 